\title{
Intelligent Control Architecture for Assistive Mobile Robots
}

\author{
Silas Franco dos Reis Alves $^{1}{ }_{(\mathbb{C})} \cdot$ Humberto Ferasoli Filho $^{2}$
}

Received: 30 November 2015 / Revised: 31 March 2016 / Accepted: 14 April 2016 / Published online: 27 April 2016

(C) Brazilian Society for Automatics-SBA 2016

\begin{abstract}
The current technology has provided the necessary means for creating better assistive tools. Among these tools, the interest on assistive robotics has been growing, since its cost is being reduced and new equipment is introduced on the market. Yet, developing robots for aiding therapy is not an easy task because robotics is intrinsically multidisciplinary. Among the several research fields contributing to robotics research, two are of particular interest: control architectures and human-robot interaction. This paper covers ongoing research projects on assistive mobile robots for child rehabilitation developed in collaboration with Brazilian and Colombian Universities and proposes an intelligent control architecture based on human-robot interaction for assistive applications. Furthermore, it presents a case study on assistive robots for special education.
\end{abstract}

Keywords Assistive robots - Social robots - Intelligent control · Intelligent systems · Human-robot interaction

This work was supported by São Paulo Research Foundation under Grants 2011/17610-0 and 2012/12050-0.

Silas Franco dos Reis Alves

salves@sc.usp.br

1 Department of Electrical and Computer Engineering, School of Engineering of São Carlos, University of São Paulo, Trabalhador São-carlense Ave., 400, São Carlos, SP 13566-590, Brazil

2 Department of Computing, Faculty of Sciences, Universidade Estadual Paulista, Engenheiro Luís Carrijo Coube Ave., 14-01, Bauru, SP 17033-360, Brazil

\section{Introduction}

Aiding people with disabilities has always been a challenging task. The current technology has provided means for enhancing existing solutions and for developing new rehabilitation or assistive tools. A current trend in assistive technologies is the usage of robots for rehabilitation (Villa-Parra et al. 2015), autism therapy (Goodrich et al. 2011; Dautenhahn and Billard 2002; Iacono et al. 2011), and social inclusion (Valadão et al. 2011). This growing interest in robotics is due to the popularization of computer devices and electronics and to the recent software tools that ease the creation of robot controllers.

Although robotics has become closer to medical applications, there are still obstacles to overcome. To researchers outside the robotics field (e.g., surgeons, psychologists, physiotherapists, and computer scientists), the cost and the experience necessary to work with robots become a barrier (Goodrich and Schultz 2007). Robots developed for a particular goal (e.g., surgical robots) are expensive and require skilled personnel, and these researchers have no access to them (Burke et al. 2004).

In the case of robots for social inclusive robots, two research fields play a major role on the final results of the robot: control architectures and human-robot interaction (HRI). While HRI studies how a robot can interpret and convey human information through verbal and nonverbal languages, control architectures determine how the many robot modules, including those for HRI, will work together in an ordered and coherent course of action to achieve a given goal.

This paper presents the ongoing experiences using affordable mobile robots for the rehabilitation and social inclusion of children with severe motor impairments and also proposes an intelligent control architecture for creating applications for this end. Thus, Sect. 2 presents the previous experiences 
with assistive robotics, whereas Sect. 3 presents the current robot solution that has been developed. In sequence, Sect. 4 introduces the intelligent control architecture, which is used to compose the assistive application described in Sect. 5. Thereafter, Sect. 6 presents the results obtained with the application. Finally, the final discussion and conclusions are presented in Sect. 7.

\section{Previous Work}

Our research in the field of rehabilitation robotics began with a collaboration project involving the Federal University of Espírito Santo UFES (Vitória, Brazil) and the State University of São Paulo UNESP (Bauru, Brazil). This project resulted in the development of two mobile robots, i.e., 14-bis and Roburguer. In the 14-bis robot illustrated in Fig. 1a, it is easy to see the center of the drawing pen and the LEDs used to indicate the robot state. In relation to the Roburguer robot presented in Fig. 1b, an aluminum structure insinuates a heavyduty robot for moving things. Both robots were used for the rehabilitation and social inclusion of children with severe motor disabilities.

In order to explore how the study of control architectures could contribute in the development of assistive robots, the subsequent collaborative project, Carmen, proposed that a mobile robot with simple autonomous behaviors could be more entertaining and friendly for children. Currently, a new robot, named Pomodoro, is being used to further explore how control architectures and HRI should be combined in order to create better robots for such cases.

\subsection{The Robots 14-bis and Roburguer}

It is known that environmental exploration and socialization are a huge obstacle for children with severe motor impair- ments. Restrained to limited life experiences, these children can develop learned helplessness, which is the belief that one is unable to do anything for oneself. To provide some autonomy and independence for these children (Valadão et al. 2011), a general-purpose robot has been adapted so they could teleoperate it by means of a hybrid sensor, developed at UFES, which combined surface electromyography (sEMG) and an accelerometer.

To improve that project, a partnership between UFES and UNESP led to the development of two affordable robots designed for ludic activity with children: 14-bis, a drawing robot, and Roburguer, a robot for moving things. Supposing that children mostly understand robots as toys, the same design rules applied to toys were applied when designing these robots.

The emotional experiences promoted by assistive toys are an important part of cognition process of children, thus affecting the robot development. As consequence, the two robots are colorful and have different shapes and purposes. In this sense, Roburguer resembles a heavy-duty robot that can move objects, providing a grabber to push and pull tiny objects around, whereas in the case of 14-bis the shape alludes to Santos Dumont's airplane-not as an attempt to honor the historical person, but to place the pen in the center of the robot and make it as visible as possible.

The diagram of Fig. 2 shows the global control system of both robots. The hybrid sensor was attached to the child's head, allowing the accelerometer to read the inclination of the head, whereas the electrodes were attached to a muscle that the child could control. With this setup, the data collected from the hybrid sensor were processed by a workstation (a standard PC), which implemented a simple behavior-based control architecture for teleoperating the robot: the head's inclination was converted into the robot's linear and angular speeds, whereas the muscle state (that could be either flexed or extended) activated the pen of 14-bis or the grab-

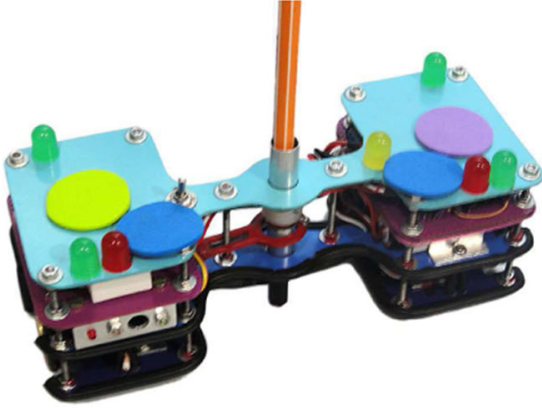

(a)

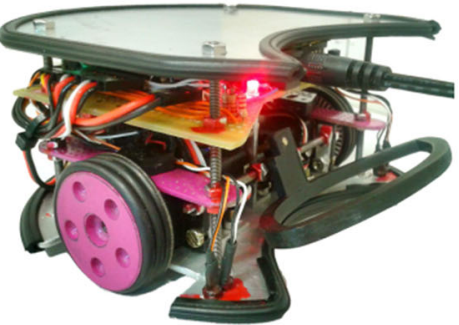

(b)

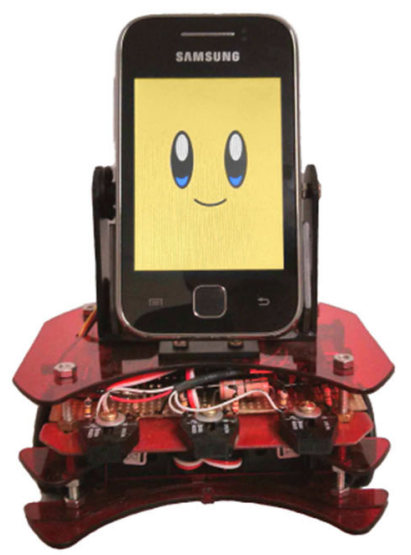

(c)

Fig. 1 The robots 14-bis (a), Roburguer (b), and adapted Roburguer(c) 


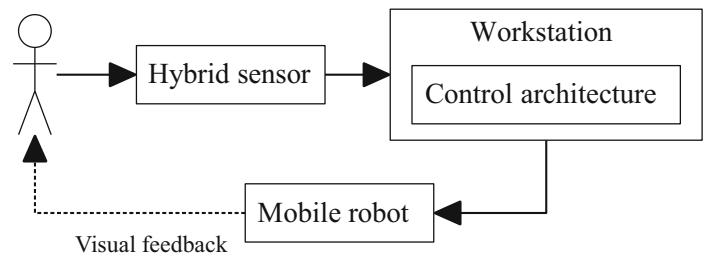

Fig. 2 Global system for 14-bis and Roburguer

ber of Roburguer. Moreover, this architecture provided three behaviors:

Wander behavior makes the robot to move around randomly and is triggered when the child does not move for a while.

Fear behavior was developed in order to keep the robot safe, hence avoiding falling from tables. It is triggered by the robots ground infrared sensors.

Hunger behavior is triggered when the robot's battery level is low. It is used to indicate that the robot has to be recharged.

\subsection{Improved Global System}

The main problems associated with the preliminary global system (Fig. 2) were the need of a workstation, which reduced the system mobility. It required time and effort to move the workstation and the robots from the laboratory to the therapy rooms. To improve the system mobility and reduce its size and cost, the workstation was replaced by an Android smartphone, as shown in Fig. 3.

The smartphones have improved their functionality, connectivity and energy efficiency, while reducing their costs. A common smartphone embeds a set of devices and interfaces that can be used for robotics, such as the processing unit, touchscreen, camera, accelerometer and gyroscope, audio input and output, and wireless communications. The chosen mobile operating system, namely Android, provides services of speech recognition and synthesis, which are used for controlling the robot by speech. Furthermore, Android is supported by a large set of free software tools and libraries that ease the creation of new applications.

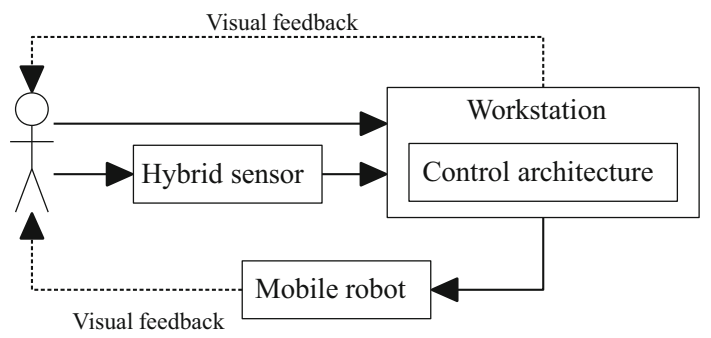

Fig. 3 Improved global system, which uses a smartphone instead of the workstation
To attach the smartphone on the robot, an articulated base was built on Roburguer, as shown in Fig. 1c. The inclination of the base is modified by a standard radio-control servomotor.

The improved global system, illustrated by Fig. 3, uses both the hybrid sensor (likewise the preliminary) and the smartphone to interact with the user. Besides acquiring data from the hybrid sensor, processing the control architecture and activating the robot, the smartphone also displayed a smiling face. If the hybrid sensor was not available, the two new behaviors would be introduced to enable the robot control by speech:

Seek faces behavior is triggered by the wander behavior. It captures image frames from the smartphone camera and extract the position of the faces, if any, by using Viola and Jones algorithm (Viola and Jones 2001) from OpenCV library. If a face is detected, it will turn the robot in that direction, so that the face is centered on the image.

Speech interaction behavior is activated by the seek faces behavior when a face is found and inhibits the seek faces and wander behaviors. When active, this behavior (using Android services) listens to the user voice commands and either executes the recognized command or says that it could not understand the user, and after this, it asks for a new command.

\subsection{Preliminary Conclusion}

In preliminary experiences, both 14-bis and Roburguer robots have been seem as attractive for children. Likewise, the proposed control architecture has increased the interaction between children and the robot, as the robot may or may not do what the children commands, thus instigating them to play with the robot to discover why. Although just a five simple autonomous behaviors have been implemented, they were enough to entertain the children and nurture their exploratory spirit.

If the assumption that robots with some degree of autonomy are more interesting for therapy than teleoperated toys, then the design of control architectures within the context of HRI is of particular importance. It is the control architecture that defines and orchestrates the harmonious operation of the different modules that compose a robotic system.

Motivated by the need of control architectures for HRI in the field of assistive robots, we propose an intelligent control architecture inspired by natural models whose main purpose is to allow the robot to interact with people intuitively to motivate the collaboration between them.

\section{Pomodoro Robot}

The positive experience with previous robots and the need of affordable and intuitive robot platforms have led to the devel- 


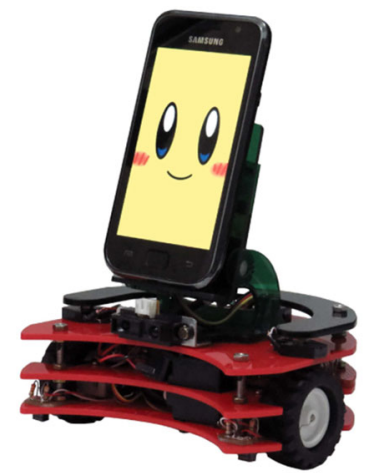

(a)

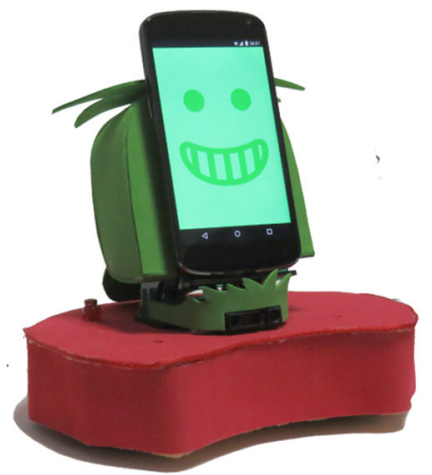

(b)
Fig. 4 Pomodoro robot (a) with costume (b)

opment of Pomodoro robot, presented in Fig. 4, which is our current base for experiments involving control architectures, HRI and assistive robotics. Pomodoro is a 100 USD robot that is easy to replicate and provides high flexibility. This robot followed the same design rules of 14-bis and Roburguer, and thus, it uses vivid colors to draw children's attention, and the body is designed with rounded shapes to not harm the user. The body structure uses flat acrylic parts that can be easily replicated.

To help the dissemination of these assistive solutions, a project in collaboration with the Virtual Reality Center of the Nueva Granada Military University, UMNG (Bogotá, Colombia), created a virtual model of Pomodoro. The virtual model can be used when the physical robot cannot be afforded, or to create Web-learning virtual environments. Pomodoro's hardware architecture is similar to the adapted Roburguer robot and thus consists of two separate modules: an integrated hardware, which runs a supervisor software, and the smartphone, which will implement the control architecture. The supervisor is responsible for reading the sensors and activating the actuators and can operate in two modes: non-autonomous (executing the smartphone's commands without any reasoning) and semi-autonomous (it requires the smartphone's commands to operate, but it has simple behavior-based control architecture to avoid falling and obstacles).

The first experience using Pomodoro was an assistive application for encouraging the motion of the upper and lower members for rehabilitation or entertainment. This application used a Leap Motion gesture tracking device for converting the user's hand gestures into commands for the robot. The hand's flexion and extension were translated into the robot linear speed, whereas the hand's radial/ulnar deviation was translated into the robot angular speed. Its setup was similar to that of Fig. 3, where the hybrid sensor was replaced by the Leap Motion, and used a behavior-based architecture using wander, fear, and hunger behaviors.

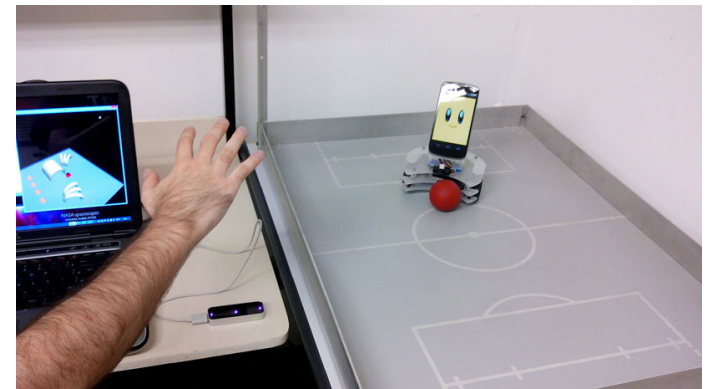

Fig. 5 User exercising his hand to control the mobile robot

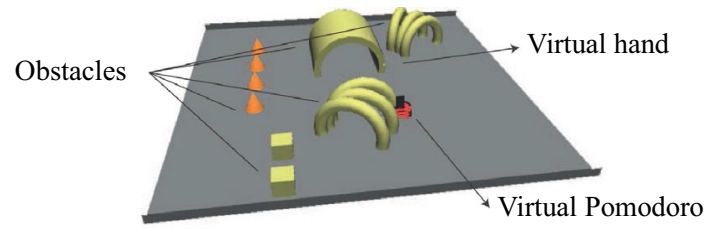

Fig. 6 Virtual environment where the user had to drive the virtual robot at a maze

This resulted in the development of two rehabilitation games: one using the real robot and the other using its virtual counterpart. Both games were developed to encourage the user to perform flexion/extension and ulnar/radial deviation with his/her hand in order to exercise this movements. The game using the physical robot was based on a soccer game (Fig. 5), where the user had to guide the robot so that it would push the ball to the goal. The other game displayed a virtual maze, and the user had to guide the virtual robot through this maze without hitting any virtual obstacle (Fig. 6). Further development will address the Leap Motion accuracy, as in (Du and Zhang 2015).

\section{Intelligent Control Architecture}

The architecture proposed in this work can be understood from two different points of views. The operational point of view organizes the architecture according to the hierarchy of the various hardware devices that composes the mobile robotic system. It determines the spatial relationship between the various modules, i.e., in which device each module is executed. On the other hand, the logical point of view is concerned with determining information flows independent of the spatial relationship between them, i.e., without regard to which device implements a given module.

\subsection{Operational Point of View}

As can be seen from Fig. 7, there are three components on the architecture: supervisor, computer, and cloud. At the lowest level of the hierarchy is the supervisor, corresponding in this 


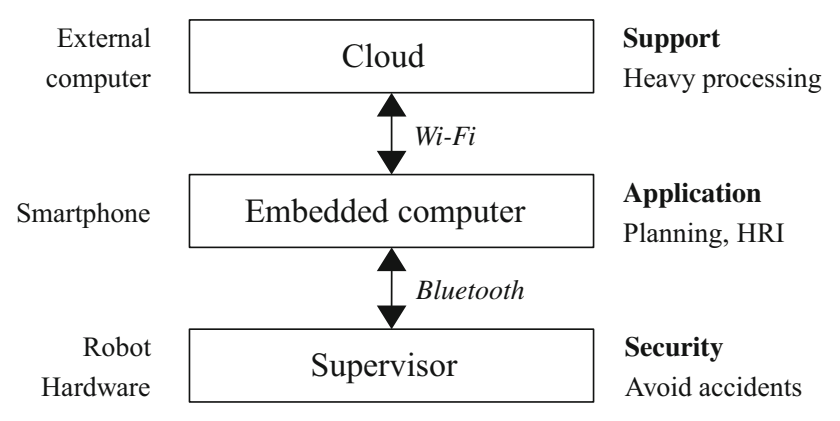

Fig. 7 Operational point of view

case to the hardware of Pomodoro robot. The supervisor, in addition to providing sensory reading and drive actuators, should provide a security layer that prevents the robot to take some actions that clearly endanger itself. Examples include situations in which the robot is on the verge of falling-its ground sensors can detect if there is a hole in front of its wheels-or collision - when its proximity sensors identify an obstacle. Such preventive action should be taken by the supervisor so that the robot can preserve its physical integrity even if the software of higher levels fails to control the robot.

At the intermediate level lies the embedded computer, responsible for implementing the decision-making algorithms of the mobile robot. In the case of Pomodoro robot, it consists of a smartphone communicating via Bluetooth with the supervisor.

There may be situations where the availability of an external computer system can increase the robot's performance, providing increased computing power, or even allowing the fulfillment of the task when it has a crucial fact unknown to the robot. The cloud layer represents these external computer systems, which support the robot with heavy processing, searching large databases (e.g., big data), or communicating with different agents via Wi-Fi.

The main motivation to analyze the control architecture from the operational point of view is to identify the potential communication bottlenecks that may compromise the robustness of the robotic system, allowing the proposition of a fault-tolerant system.

\subsection{Logical Point of View}

The logical viewpoint is concerned exclusively with the flow of information, giving little attention to structural issues of the system hardware. To enable a rich and meaningful interaction for problem solving, the control architecture requires means to enable autonomy, teamwork, communication with other agents - humans or otherwise - and learning. At the same time, the architecture must provide the algorithms that solve the remaining challenges of robotics, such as navigation. In order to address these problems and chal- lenges, this project proposes a hybrid, bio-inspired control architecture composed of both deliberative and reactive modules that mimic the elements found in animals to provide intelligence - albeit limited— to the robot.

Other distinguishing feature of the proposed intelligent control architecture is its distributed nature. As will be described in the next sections, each module consists on an independent software application. This allows the architecture to exploit the parallel computing capabilities of the hosting machine, reduces the scope of software failures, and creates a flexible environment where modules can be added to the system without the need of recompiling existent modules.

The intelligent control architecture is composed of four main modules, as shown in Fig. 8. The memory space is the central module of the architecture, providing means of interprocess communication and data storage. The acquisition of sensor data, as well as the low-level control of actuators, is performed by the hardware interfaces. The data processors implement different services of data processing. The controller is the software that effectively controls the robot. The next sections will address the design and development of each module.

\subsubsection{Memory Space}

The memory space has two objectives: to serve as a communication mean and to store data. It is inspired on the psychological concept of modal memory (Atkinson and Shiffrin 1968), which defines the human memory in three types:

Sensory memory captures each sensory stimuli, which will be forgotten if it receives no attention.

Short-term memory also known as working memory. It stores the relevant stimulus and information regarding a current task and needs to be rehearsed in order to not be forgotten.

Long-term memory stores a set of organized information for a undetermined time.

To compose the memory space with such features, a whiteboard system (Reilent 2012; Thórisson et al. 2005) was implemented using the NoSQL (not only structured query language) database Redis. An important feature of this database is that the user can specify for how much time a given data will be stored before being deleted. This is called the time to live (TTL).

\subsubsection{Hardware Interfaces}

The hardware interfaces are the modules that capture and decode sensory data and that drive the actuators. Thus, the 


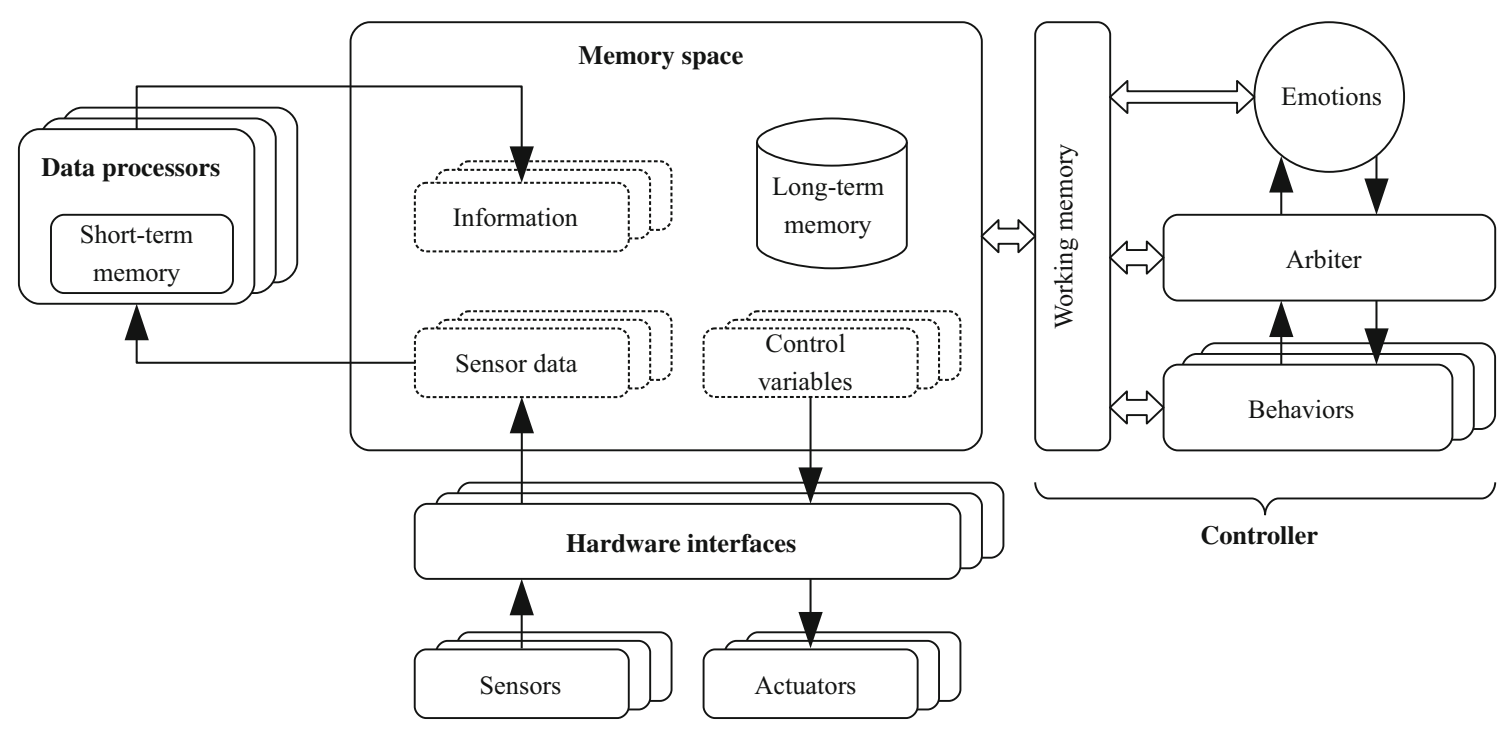

Fig. 8 Intelligent control architecture

hardware interfaces "produce" decoded sensor data at the memory space with a short TTL, usually $1 \mathrm{~s}$, and "consume" information about the set points of its actuators.

\subsubsection{Data Processors}

Data processors are responsible for reading and processing decoded sensory data to produce useful information to the system. The definition of these modules is wide as they have the purpose of providing data-processing services to the controller, thereby reducing the complexity of the controller.

\subsubsection{Controller}

The controller is the module that effectively controls the mobile robot. In this architecture, it is composed of three layers, as shown in Fig. 8, namely emotion, arbiter, and behaviors.

Emotion The addition of artificial emotion intends to improve the relation between human and robot, since HRI is an important feature of this intelligent control architecture, and emotions plays a essential role in human communication and decision taking. For psychology, emotion is generally understood as feelings that involve subjective appraisal, physiological processes and cognitive beliefs (Gazzaniga et al. 2012)_although there is not a consensual definition of "emotion" (Mulligan and Scherer 2012). Emotions are considered as response of short duration to a given stimulus, unlike humor, which have longer duration (Gazzaniga et al. 2012).

The two main dominant psychological theories on emotion are appraisal theories and dimensional theories. The artificial emotion was designed using the circumplex model (Posner et al. 2005; Russell 1980), a dimensional theory where emotions are defined by its arousal and valence into a continuous, non-discrete dimensional space (Larue et al. 2013), illustrated by the chart of Fig. 9, where $\alpha_{0}, \alpha_{1}, \ldots, \alpha_{9}$ is defined by the sequence given by 1 . The Arousal axis indicates the level of enthusiasm or agitation, whereas the valence axis specifies how pleasant, or unpleasant, the experience is. The original model places 28 emotions along both axes; however, for the sake of simplicity, they were discretized to 11 emotion "sections." The emotions happy, sad, disgust, fear, anger, and surprise are analogous to the six "universal emotions" of ( Ekman 1999). The emotions excited and calm are considered, respectively, as "high-aroused" and "low-aroused" happiness - the same way that depressed is a "low-valence" sadness. The sleepy emotion indicates fatigue. The emotion is classified as neutral when its magnitude is smaller than the threshold $\mathrm{N}_{\mathrm{m}}$, specified in Fig. 9. Additionally, in this architecture, the robot artificial humor is considered as a permanent emotion which varies with time, as the robot experiences different emotions during operation.

$\left(50^{\circ}, 20^{\circ}, 340^{\circ}, 300^{\circ}, 240^{\circ}, 195^{\circ}, 160^{\circ}, 140^{\circ}, 110^{\circ}, 80^{\circ}\right)$

Knowing that the arousal $a$ and valence $v$ are defined within $[-1,1]$, the magnitude and angle of the emotion given by $e=(a, v)$ can be, respectively, expressed as given by Eq. 2 .

$e_{\mathrm{m}}=\sqrt{a^{2}+v^{2}} \quad \alpha_{e}=\arctan \frac{a}{v}$ 


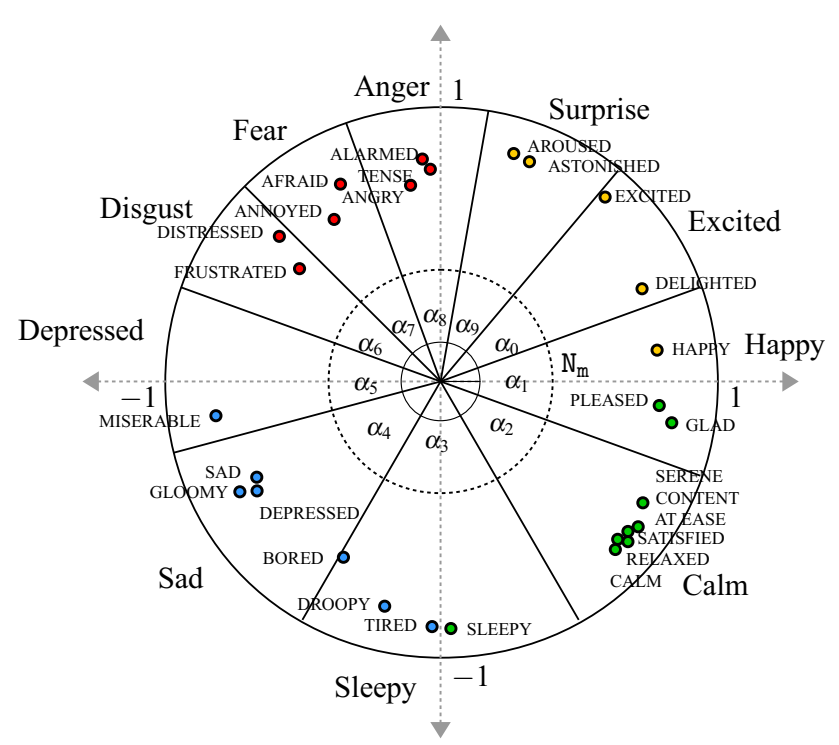

Fig. 9 Adapted circumplex model

Henceforth, if $e_{\mathrm{m}}<\mathrm{N}_{\mathrm{m}}$, then the emotion is neutral; otherwise, the emotion can be classified by the value of $\alpha_{e}$ according to the sections of Fig. 9.

Similar approaches are found in the literature. In (Han et al. 2013; Zhang and Sharkey 2012), the circumplex model is used to generate the expression of a humanoid animatronics, while in (Sellers 2013) this model is extended to several two-dimensional planes.

Behaviors Behavior-based control architectures have been studied for 30 years and provide a well-defined framework for creating both reactive and hybrid architectures. Behaviors are the building blocks of these control architectures, being analogous to a state of a finite state machine (FSM). In fact, using FSMs for defining a control architecture is convenient because it allows the formal analysis of the system by the means of a high-level language (Arkin 1988). In addition, the motor schema model (Arkin 1989) was employed to design the behaviors.

Each time a stimulus triggers a behavior or a transition within the FSM of a behavior, it also triggers a predetermined artificial emotion with a given magnitude. The triggered emotion will then update the humor of the robot. If the emotion magnitude is strong enough, it may cause the robot to perform an impromptu short-duration reaction, e.g., displaying a scared face, even with a calm humor.

Arbiter Unforeseen real-world scenarios can compromise the security or the accomplishment of the task. In such cases, the planning may need to be updated or a special behavior must be triggered. The arbiter is the module that orchestrates the behaviors so that the robot achieves its goals.

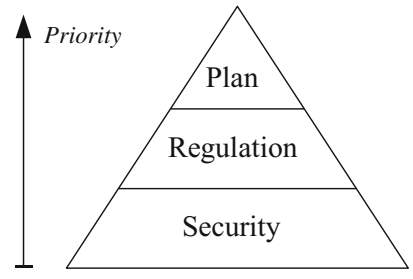

Fig. 10 Mobile robot needs

Since the arbiter should make decisions under different circumstances-sometimes with conflicting objectives-it is evident the need to organize the objectives to be met. Inspired by Maslow's hierarchy of needs (Maslow 1943), which defines a hierarchy of human needs, a pyramid of needs with three hierarchical layers was designed, as shown by Fig. 10. The three layers that enable the satisfactory operation of the mobile robot are the following:

Security is the layer on the base of the pyramid, meaning that any behavior triggered for security reasons have priority over the others, and will be executed until the robot is out of danger.

Regulation is inspired by the physiological concept of homeostasis. Homeostasis is the ability of a biological system to maintain stable and relatively constant their internal conditions by means of regulating mechanisms (Jakoi and Carbrey 2015). For a robotic system, regulation is a set of processes that maintain internal integrity and operability of the robot. The processes of this layer intend to, for example, keep the battery charge at secure levels, keep the load on actuators in a safe range, and check the system for faults.

Plan is the layer which is concerned with the global objective of the robot and the set of actions needed to reach it. The plans can be generated by a planner algorithm or predefined by a specialist.

\section{Assistive Social Robots}

The literature has reported positive results in the employment of assistive mobile robots in the therapy of children diagnosed with autism spectrum disorder (ASD) (Robins et al. 2005; Duquette et al. 2007; Robins et al. 2010; Coeckelbergh et al. 2015; Kim et al. 2012; Iacono et al. 2011). It suggests that the artificial and predictable nature of robots is easier to understand and more stimulating for children with ASD (Duquette et al. 2007).

In order to stimulate social skills in children, an application of social interaction with robots was proposed. In this application, a child can interact with the mobile robot as if it was an "electronic pet." When the robot gets the child attention, it tries to interact with him or her using ludic activities. 
To develop such application, the intelligent control architecture was used. Hence, two hardware interfaces were developed, one for the body of the robot and other for the smartphone, besides one data processor for detecting faces (with OpenCV library) and QR codes (with ZXing library).

The next subsections will address the behavior of each layer of the arbiter, which are illustrated by FSMs, or motor schemata in cases where a FSM would have a single state. It is important to highlight that even though each sub-behavior, i.e., state of a FSM, is ultimately described as a motor schema, not all motor schemata will be showed for concision.

\subsection{Plan Layer}

The plan layer has the behaviors that implements the games card, dance, and touch, and also a behavior that allows the robot to move over the workspace searching for a personthus search person.

At the beginning of the therapy, the robot will either search for a person or will dance to draw attention to it and will keep doing that until it finds someone, or until it becomes tired, sad, or depressed. When if finally finds a person, the robot will trigger a game at random. If it loses sight of the person, it will go back to search for a person and dancing.

It is important do stress that when a face is detected by the camera of robot, it will move the servomotors of its "head" so that it is always "looking" at the user face-thus implementing the behavior of social gaze (Emery 2000).

Search person The robot moves freely within the desktop in search of a person using face detection, as shown in Fig. 11. If more sensors were available, it would be possible to combine them as in (Pereira et al. 2013).

Dance play The robot invites the child to dance, while the robot plays a song and moves, as shown in Fig. 12. The purpose of this activity is to develop the ability of the child to pay attention to verbal commands and also to use his or her body to express himself or herself.

Card play The robot invites the child to play a game whose goal is to teach the child some basic concepts using picture

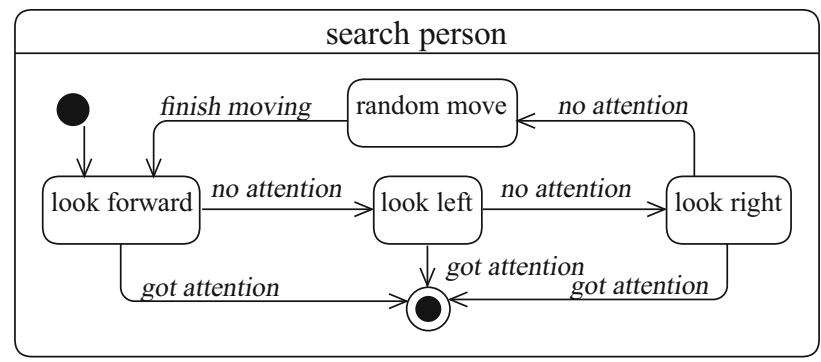

Fig. 11 FSM of the behavior Search Person

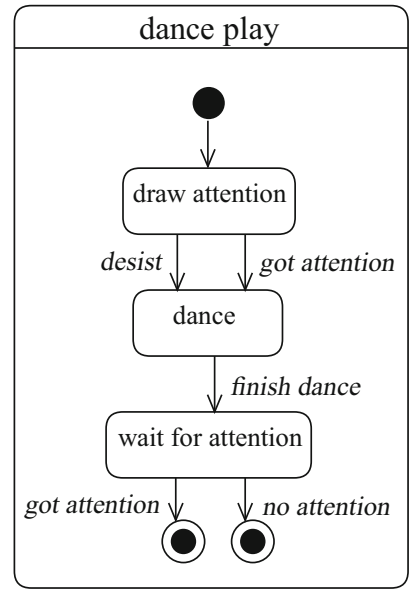

Fig. 12 FSM of the behavior Dance Play

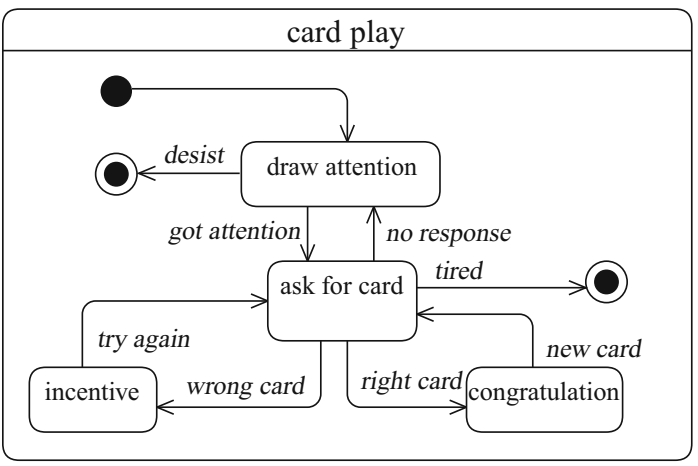

Fig. 13 FSM of the behavior Card Play

cards. To help the robot to identify the cards, a QR code with the picture's name is printed on each one. With the picture cards placed on the table, the robot shows one of the pictures asks the child shows that card. If the child does not show a card, the robot retries asking for a given number of times. If the child shows the wrong card, the robot encourages the child to keep trying, and him or her show the right card, the robot congratulates. The FSM of this behavior is shown in Fig. 13.

Touch play The robot asks the child to touch its face, as shown in Fig. 14. Though simple, this activity aims to reduce the aversion for touch felt by children with ASD.

\subsection{Regulatory Layer}

The regulatory layer in this application is activated whenever the robot is tired, sad, or depressed with $e_{\mathrm{m}}>0.75$. In such cases, the behavior sleep will be triggered, so that the robot can rest to "recover." This layer could have more complex behaviors and rules; however, the application does not require such behaviors, and the robot sensors are too lim- 


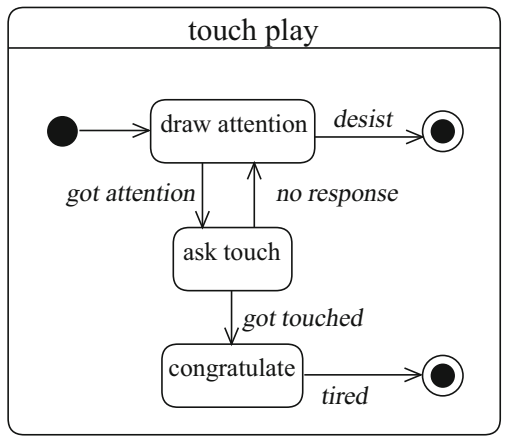

Fig. 14 FSM of the behavior Touch Play

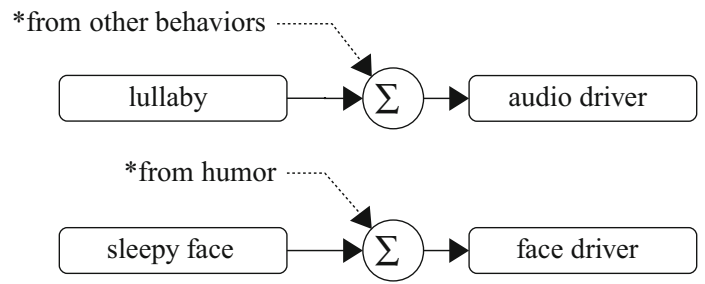

Fig. 15 Sleep motor schema

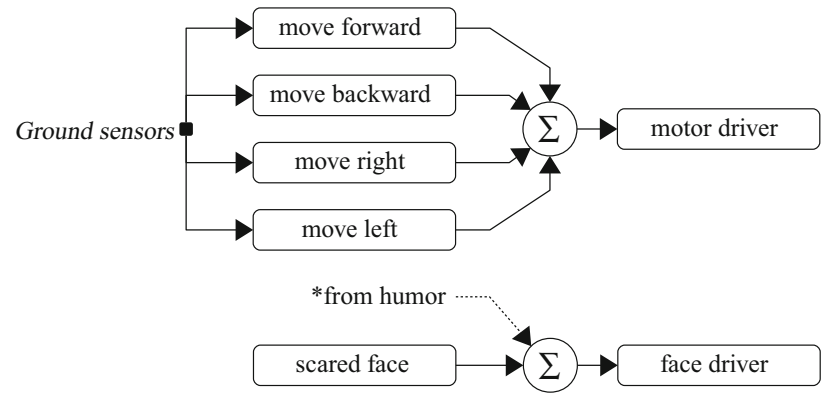

Fig. 16 Avoid Danger motor schema

ited to propose them. Henceforth, in the sleep motor schema, shown in Fig. 15, the robot pretends to be asleep.

\subsection{Security Layer}

The security layer only intended to avert falling due to restrictions of the sensors of the robot. To this end, the avoid danger behavior, illustrated as a motor schema by Fig. 16, drives its actuators to ensure its physical integrity and user safety. For Pomodoro robot, this means it will use its infrared ground sensor to avoid falling.

\section{Results}

In order to verify the application, a mock therapy session was conducted in the laboratory, where an adult volunteer played the role of the child. The workspace was a table with white tabletop, whose extremities were covered with a black adhesive tape. The black tape allowed the robot to detect the limits of the workspace using the IR ground sensors. The environmental lightening was also controlled to provide better image quality.

To reduce the duration of the experiments, the initial humor and the emotion magnitudes were adjusted to make the robot more "sensitive." Therefore, the initial humor was given $v=0.4$ and $a=0$, with $\mathrm{N}_{\mathrm{e}}=0.25$, thus being classified as happy.

\subsection{Experiment 1: Regular Behavior}

This experiment was conducted to demonstrate the regular behavior of the robot during a therapy session. To this end, an adult volunteer played the role of the child and interacted with the robot. This section describes this experiment.

As discussed in Sect. 5.1, the behavior search person is triggered, and thus, the robot starts moving in search of a person. As the robot wanders without finding anyone, its valence and arousal drops, causing the humor to become sad around $20 \mathrm{~s}$, as shown in Fig. 17. When a person is finally found at $36 \mathrm{~s}$, the card game behavior is triggered and the humor improves.

However, during the execution of card game the volunteer looked away, and thus, his face was not detected and the search person was triggered again at $46 \mathrm{~s}$, as seen in Fig. 17. After finding his face, the behavior card game is resumed at $49 \mathrm{~s}$. During the game, the volunteer answered correctly the robot questions using the cards; hence, the humor of the robot evolved from happy to excited.

At $73 \mathrm{~s}$, the volunteer moved, loosing visual contact with the robot again. As a result, the robot suddenly returned its "head" to its initial position, causing the body of the robot to slope for a short time. Although short, this time was enough to trigger the avoid danger for approximately $0.5 \mathrm{~s}$. Soon after that, search person was triggered and found the face of the volunteer while he was still moving. Because of that, the touch play was started at $77 \mathrm{~s}$ and interrupted at $79 \mathrm{~s}$ to trigger, again, the search person behavior. This caused a minor effect on the humor, where $a$ had a small decrease, while $v$ was almost stable, meaning that the robot got "tired," but did not experience an "unpleasant" experience.

This time, however, the robot moved over the workspace limits, causing the it to activate the avoid danger behavior until the robot got to a safe position, at $85 \mathrm{~s}$, when the touch play behavior was resumed. Thereafter, the volunteer touched the face of the robot when asked, finishing successfully touch play at 100 s. As illustrated by the composite graph of Fig. 17, the touch play influenced positively both $a$ and $v$ values.

From $100 \mathrm{~s}$ to $176 \mathrm{~s}$, the volunteer played with the robot, which was exhibiting the card play behavior. During the game, the volunteer purposely showed two wrong cards, then four correct cards, and finally two wrong cards. Thus, it is possible to perceive a decrease on the humor from $108 \mathrm{~s}$ to 


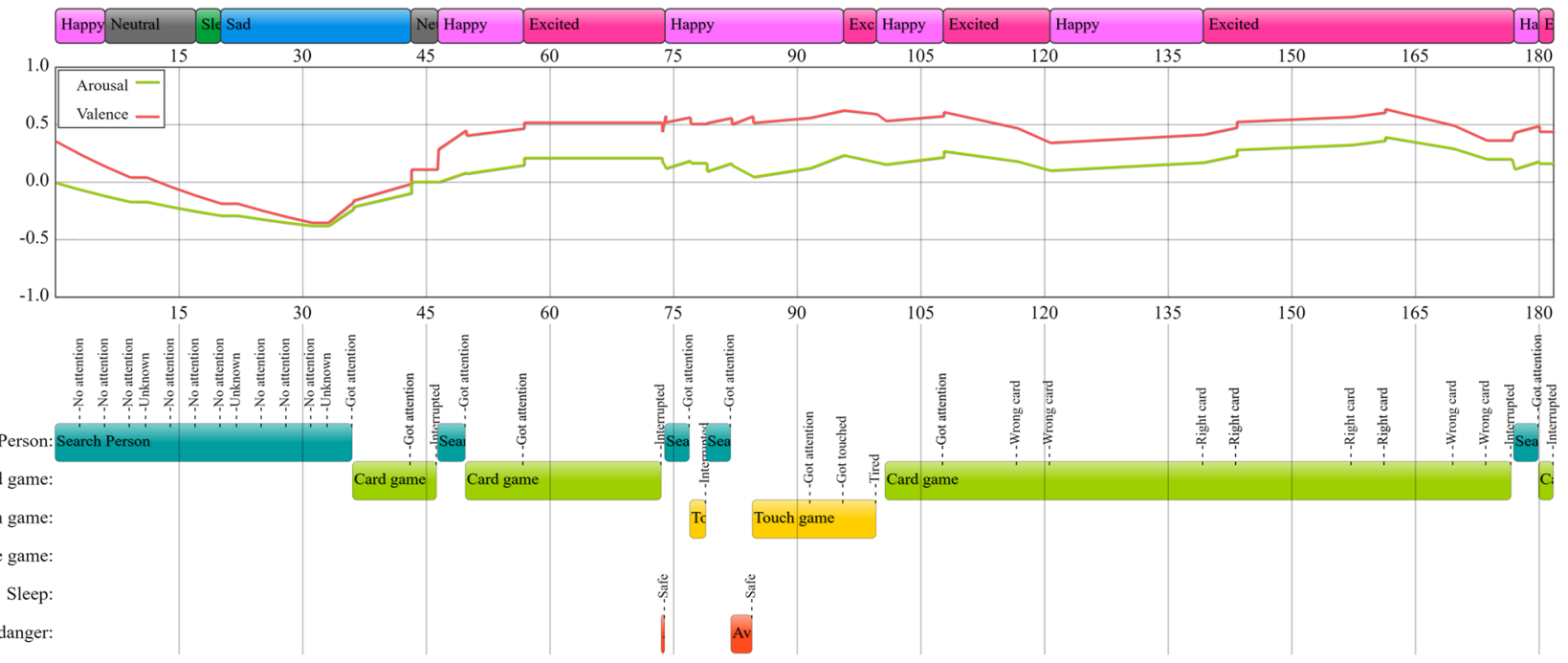

Fig. 17 Results of a mock session of therapy

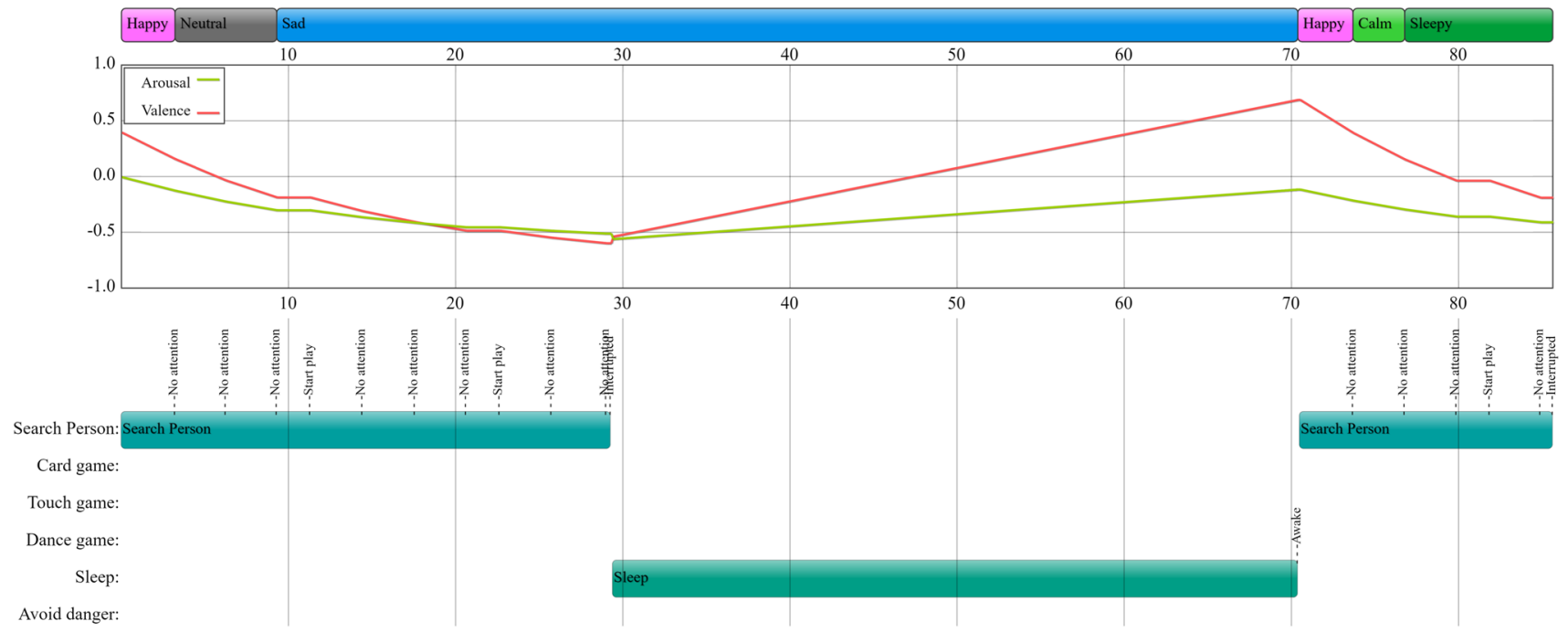

Fig. 18 Results of a mock session of therapy

$121 \mathrm{~s}$, as shown on Fig. 17. The humor improved when the volunteer showed the correct cards from $121 \mathrm{~s}$ to $163 \mathrm{~s}$, and dropped with the last two wrong cards from $168 \mathrm{~s}$ to $178 \mathrm{~s}$.

At the end, the volunteer adjusted himself in the chair, activating the search person behavior until $180 \mathrm{~s}$, when the mock therapy session was stopped.

This experiment demonstrated that the robot performed according to the requirements of the specialist team. The search person behavior was able to explore the workspace to find a person that could move during the experiment. The card play and touch play behaviors were conducted as specified by the therapists, promoting the interaction with the volunteer. The avoid danger behavior was able to keep the robot away from the workspace limits, thus ensuring its physical safety. Even when triggered by mistake, as happened at $73 \mathrm{~s}$ (Fig. 17), it did not affect negatively the robot overall behavior. Although it was not presented on this experiment, the dance play behavior also presented satisfactory results, as it was able to move the robot while playing music.

\subsection{Experiment 2: Coping Behavior}

The goal of this experiment is to demonstrate how the absence of social interaction affects the humor and global behavior of the robot. Thus, there was no volunteer to interact with the robot.

As the robot could not find the volunteer, the robot triggered the behavior search person, thus driving the robot over the workspace in search of the volunteer. Due to the absence of interaction with people, the robot starts experiencing "sad" emotions, which reduces both its arousal and valence, as shown in Fig. 18. Around 30 s, the arousal and valence drive 
the robot's humor to an undesirable state, as can be seen in the composite graph of Fig. 18. This causes the deliberator to stop the behavior search person and trigger the behavior rest. As the robot rests, the values of both arousal and valence increase; hence, when the robot "awake" at $70 \mathrm{~s}$, its humor is reclassified as "happy," as shown in Fig. 18. After that, the robot triggers the behavior search person and restarts the loop.

The composite graph of Fig. 18 shows that, as expected, the robot's humor had a negative change while being ignored, leading it to a lethargic behavior. This conduct is similar to that observed in individuals which employ "resting" as a coping behavior. It is imperative to observe that the goal of this experiment was to reveal that the robot behavior is analogous to that found in animals, and not to prove that its inner structures are similar to their biological counterpart.

\section{Conclusion}

The use of assistive mobile robots is increasing, since robotics is becoming more available and easy to use. Since these robots must interact with people, both patient and the therapist, they will need well-designed HRI. Moreover, the robots will require a control architecture that allows them to perform their tasks in a human-centered environment.

Motivated by the need of control architectures for HRI in the field of assistive robots, the intelligent control architecture was proposed and developed. It was inspired by natural models whose main purpose is to allow the robot to interact with people intuitively to motivate collaboration between them. Hence, the intelligent control architecture includes aspects related to the task and HRI inspired by biological sciences and psychology to propose mechanisms that emulate emotions.

The latest Pomodoro robot has been designed in order to contribute to the area of HRI with a robotic system that is compact and inexpensive to replicate, allowing its adoption for research and entertainment. A virtual model of Pomodoro was also created, so that it can be used even when the real robot is not available.

To evaluate the intelligent control architecture, an assistive application was designed. In this application, the robot would play with a child while displaying faces according to its artificial humor and emotions. As presented in Sect. 6, the robot performed as expected and fulfilled its goal of interacting with the volunteer encouraging him to play. The dynamical facial expressions, which changed according to the humor of the robot, allowed the robot to express nonverbal information about its internal state. Henceforth, the proposed framework for handling emotions also proved efficient, since it could imitate human emotion with little computing. Certainly, since the controller lacks a method for learning the relationship between behavior and emotions, the robot designer must define them when projecting the behaviors. Namely, it is the robot designer who embeds the cognitive beliefs of emotion into the transitions of the robot's behavior.

The intelligent control architecture was built with a software architecture that allowed the quick development and test of software. By separating each software as a standalone module, it was possible to test them isolated from the system. The adopted database, Redis, eased the development of such features, since it is designed for fast information retrieval.

Whereas the use of synthetic emotions can help the system to adjust or change its behavior, it is true that it can also introduce uncertainties into the decision making. For example, if the robot humor is classified as "fear" with high magnitude, it can avoid triggering the correct behavior in order to ensure its safety. This would characterize an improper coping behavior, similar to that of the experiment of Sect. 6.2. The robot designer thence must project the behaviors of regulatory layer in order to implement proper coping behavior for the robot. Additionally, more refined methods for decision making that inherently handle uncertainties, such as fuzzy systems, could be adopted to improve the robot global behavior.

Besides improving the controller with sophisticated decision-making algorithms, future works also include exploiting the current NoSQL database in order to improve the memory system. Because it does not require a static schema, such as relational databases that relies in well-defined tables, it is possible to modify the recorded data structures during operation time. This could be used to enable learning algorithms or to implement episodic memory for the robot.

Acknowledgments We thank the Group of Integration of Intelligent Systems and Devices (GISDI), of the Department of Computing, Faculty of Sciences, São Paulo State University, Bauru, Brazil. We also thank the Laboratory of Intelligent Automation (LAI), of the Department of Electrical Engineering, Center for Technology, Federal University of Espírito Santo, Vitória, Brazil, for their support. We also thank Freepik for the pictographs and to Kevin MacLeod for the music.

\section{References}

Arkin, R. C. (1988). Behavior-based robotics. Cambridge: MIT Press. Arkin, R. C. (1989). Motor schema-based mobile robot navigation. The International Journal of Robotics Research, 8(4), 92-112.

Atkinson, R. C., \& Shiffrin, R. M. (1968). Human memory: A proposed system and its control processes. The Psychology of Learning and Motivation, 2, 89-195.

Burke, J., Murphy, R., Rogers, E., Lumelsky, V., \& Scholtz, J. (2004). Final report for the DARPA/NSF interdisciplinary study on human-robot interaction. IEEE Transactions on Systems, Man and Cybernetics, Part C (Applications and Reviews), 34(2), 103-112.

Coeckelbergh, M., Pop, C., Simut, R., Peca, A., Pintea, S., David, D., et al. (2015). A survey of expectations about the role of robots in robot-assisted therapy for children with ASD: Ethical acceptability, trust, sociability, appearance, and attachment. Sci- 
ence and Engineering Ethics, 22(1), 47-65. doi:10.1007/s11948015-9649-x.

Dautenhahn, K., \& Billard, A. (2002). Games children with autism can play with robota, a humanoid robotic doll. In S. Keates, P. Langdon, P. J. Clarkson, \& P. Robinson (Eds.), Universal Access and Assistive Technology: Proceedings of the Cambridge Workshop on UA and AT 'O2 (pp. 179-190). London: Springer London. doi:10. 1007/978-1-4471-3719-1_18.

Duquette, A., Michaud, F., \& Mercier, H. (2007). Exploring the use of a mobile robot as an imitation agent with children with lowfunctioning autism. Autonomous Robots, 24(2), 147-157.

Du, G., \& Zhang, P. (2015). A markerless human-robot interface using particle filter and kalman filter for dual robots. IEEE Transactions on Industrial Electronics, 62(4), 2257-2264.

Ekman, P. (1999). Handbook of cognition and emotion. In T. Dalgleish \& M. J. Power (Eds.), Basic emotions (pp. 45-60). John Wiley \& Sons, Ltd.

Emery, N. J. (2000). The eyes have it: The neuroethology, function and evolution of social gaze. Neuroscience and Biobehavioral Reviews, 24(6), 581-604.

Gazzaniga, M., Heatherton, T., \& Halpern, D. (2012). Motivation and emotion. In Psychological science (4th ed.). New York, NY: W.W. Norton \& Company.

Goodrich, M. A., Colton, M. A., Brinton, B., \& Fujiki, M. (2011). A case for low-dose robotics in autism therapy. In Proceedings of the 6th international conference on Human-robot interaction (pp. 143-144).

Goodrich, M. A., \& Schultz, A. C. (2007). Human-robot interaction: a survey. Foundations and Trends in Human-Computer Interaction, 1(3), 203-275.

Han, M. J., Lin, C. H., \& Song, K. T. (2013). Robotic emotional expression generation based on mood transition and personality model. IEEE Transactions on Cybernetics, 43(4), 1290-1303.

Iacono, I., Lehmann, H., Marti, P., Robins, B., Dautenhahn, K. (2011). Robots as social mediators for children with Autism-A preliminary analysis comparing two different robotic platforms. In 2011 IEEE International Conference on Development and Learning (ICDL) (Vol. 2, pp. 1-6).

Jakoi, E., \& Carbrey, J. (2015). Introductory Human Physiology. Lulu.com. http://www.lulu.com/shop/emma-jakoi-and-jennifercarbrey/introductory-human-physiology/ebook/product22080962.html.

Kim, E., Paul, R., Shic, F., \& Scassellati, B. (2012). Bridging the research gap: Making HRI useful to individuals with autism. Journal of Human-Robot Interaction, 1, 26-54.

Larue, O., Poirier, P., \& Nkambou, R. (2013). The emergence of (artificial) emotions from cognitive and neurological processes. Biologically Inspired Cognitive Architectures, 4, 54-68.

Maslow, A. H. (1943). A theory of human motivation. Psychological Review, 50(4), 370-396.
Mulligan, K., \& Scherer, K. R. (2012). Toward a working definition of emotion. Emotion Review, 4(4), 345-357.

Pereira, F. G., Vassallo, R. F., \& Salles, E. O. T. (2013). Human-robot interaction and cooperation through people detection and gesture recognition. Journal of Control, Automation and Electrical Systems, 24(3), 187-198.

Posner, J., Russell, J. A., \& Peterson, B. S. (2005). The circumplex model of affect: An integrative approach to affective neuroscience, cognitive development, and psychopathology. Development and Psychopathology, 17(3), 715-734.

Reilent, E. (2012). Whiteboard architecture for the multi-agent sensor systems. Doctoral thesis, Tallinn University of Technology, Tallinn, Estônia.

Robins, B., Dautenhahn, K., Nehaniv, C. L., Mirza, N. A., François, D., $\&$ Olsson, L. (2005). Sustaining interaction dynamics and engagement in dyadic child-robot interaction kinesics: Lessons learnt from an exploratory study. In Robot and Human Interactive Communication, 2005. ROMAN 2005. IEEE International Workshop on (pp. 716-722).

Robins, B., Ferrari, E., Dautenhahn, K., Kronreif, G., Prazak-Aram, B., Gelderblom, G. J., et al. (2010). Human-centred design methods: Developing scenarios for robot assisted play informed by user panels and field trials. International Journal of Human-Computer Studies, 68(12), 873-898.

Russell, J. A. (1980). A circumplex model of affect. Journal of Personality and Social Psychology, 39(6), 1161-1178.

Sellers, M. (2013). Toward a comprehensive theory of emotion for biological and artificial agents. Biologically Inspired Cognitive Architectures, 4, 3-26.

Thórisson, K. R., List, T., Pennock, C., \& DiPirro, J. (2005). Whiteboards: Scheduling blackboards for semantic routing of messages and streams. In AAAI-05 Workshop on Modular Construction of Human-Like Intelligence (pp. 8-15).

Valadão, C., Bastos, T., Bor̂tole, M., Perim, V., Celino, D., Rodor, F., et al. (2011). Educational robotics as a learning aid for disabled children. In Biosignals and Biorobotics Conference (BRC), 2011 ISSNIP (pp. 1-6).

Villa-Parra, A. C., Broche, L., Delisle-Rodríguez, D., Sagaró, R., Bastos, T., \& Frizera-Neto, A. (2015). Design of active orthoses for a robotic gait rehabilitation system. Frontiers of Mechanical Engineering, 10(3), 242-254.

Viola, P., \& Jones, M. (2001). Rapid object detection using a boosted cascade of simple features. In Proceedings of the IEEE Computer Society Conference on Computer Vision and Pattern Recognition, CVPR (Vol. 1, pp. I-511-I-518).

Zhang, J., \& Sharkey, A. J. C. (2012). It's not all written on the robot's face. Robotics and Autonomous Systems, 60(11), 1449-1456. 